\title{
G. G. Akniyev
}

\section{Approximation properties of Fourier sums for $2 \pi$-periodic piecewise linear continuous functions}

In various areas of applications the problem of approximation of continuous function $f=f(x)$, whose values are known in nodes of some grid $\Omega_{m}=$ $\left\{\xi_{i}\right\}_{i=0}^{m}$ arises. Usually, to solve this problem the polynomial spline $l_{m}^{r}(x)$ of given degree $r$ is used [1,2,3], which in simplest case $r=1$ is polyline $l_{m}=l_{m}(x)=l_{m}^{1}(x)$, that coincides in grid nodes with function $f$. If we want to store this polyline, we should store all the pairs $\left(\xi_{0}, y_{0}\right), \ldots,\left(\xi_{m}, y_{m}\right)$, where $y_{i}=f\left(\xi_{i}\right)(i=0, \ldots, m)$, which may take o lot of storage space if number of nodes is big. In this connection the interim problem of compression of information $\left(\xi_{0}, y_{0}\right), \ldots,\left(\xi_{m}, y_{m}\right)$ so that we can restore original polyline with given precision arises. To solve such problems the so called spectral method is usually applied, which is based on expansion of function $l_{m}$ in a series of a given orthonormal system and saving a minimal amount of the obtained expansion coefficients provided an ability to restore this function with given precision. In the present paper we attempted to solve this problem for $2 \pi$-periodic continuous polylines by expansion them into trigonometric Fourier series.

Bibliography: 9 items.

В различных областях приложений встречается задача приближения непрерывной функции $f=f(x)$, значения которой известны в узлах некоторой сетки $\Omega_{m}=\left\{\xi_{i}\right\}_{i=0}^{m}$. Наиболее часто для решения этой задачи применяют полиномиальный сплайн $l_{m}^{r}(x)$ заданной степени $r$, который в простейшем случае $r=1$ представляет собой ломаную $l_{m}=l_{m}(x)=l_{m}^{1}(x)$, совпадающую в узлах сетки $\Omega_{m}$ с самой функцией $f$. В случае, когда количество узлов сетки велико, для хранения полученной ломаной $l_{m}$ требуется запомнить большой объём информации: $\left(\xi_{0}, y_{0}\right), \ldots,\left(\xi_{m}, y_{m}\right)$, где $y_{i}=f\left(\xi_{i}\right)(i=0, \ldots, m)$, в связи с чем возникает промежуточная задача о сжатии указанной информации таким образом, чтобы ломаную можно было восстановить в последующем с заданной точностью. Для решения этой задачи, как правило, применяют так называемый спектральный метод, основанный на разложении ломаной $l_{m}$ в ряд по выбранной ортонормированной системе и хранении минимального количества коэффициентов полученного разложения, которое обеспечивает восстановление $l_{m}$ с заданной точностью. В настоящей работе предпринята попытка решить эту задачу для $2 \pi$-периодических непрерывных ломаных путём их разложения в тригонометрический ряд Фурье.

Библиография: 9 названий.

Keywords: Fourier sums, polyline, function approximation.

Ключевые слова: суммы Фурье, ломаная, приближение функций. 


\section{Introduction}

In various areas of applications the problem of approximation of continuous function $f=f(x)$, whose values are known in nodes of some grid $\Omega_{m}=\left\{\xi_{i}\right\}_{i=0}^{m}$ arises. Usually, to solve this problem the polynomial spline $l_{m}^{r}(x)$ of given degree $r$ is used [1,2,3], which in simplest case $r=1$ is polyline $l_{m}=l_{m}(x)=l_{m}^{1}(x)$, that coincides in grid nodes with function $f$. If we want to store this polyline, we should store all the pairs $\left(\xi_{0}, y_{0}\right), \ldots,\left(\xi_{m}, y_{m}\right)$, where $y_{i}=f\left(\xi_{i}\right)(i=0, \ldots, m)$, which may take o lot of storage space if number of nodes is big. In this connection the interim problem of compression of information $\left(\xi_{0}, y_{0}\right), \ldots,\left(\xi_{m}, y_{m}\right)$ so that we can restore original polylines with given precision arises. To solve such problems the so called spectral method is usually applied, which is based on expansion of function $l_{m}$ in a series of a given orthonormal system and saving a minimal amount of the obtained expansion coefficients provided an ability to restore this function with given precision. In the present paper we attempted to solve this problem for $2 \pi$-periodic continuous polylines by expansion them into trigonometric Fourier series.

This problem, in its turn, leads to question about research of approximation properties of Fourier sums for piecewise linear functions. The last question is studied good enough for smooth functions in many works, for example we refer $[4,5,6,7,8]$. In the present article we will consider approximation of continuous polylines, which are special case of piecewise smooth functions, with partial Fourier sums.

Let's proceed to a more detailed formulation of the problem. Let $\Omega_{m}=\left\{\xi_{i}\right\}_{i=0}^{m}$ be a grid of nodes $-\pi=\xi_{0}<\xi_{1}<\ldots<\xi_{m}=\pi$, and corresponding measurements are $y_{0}, y_{1}, \ldots, y_{m}=y_{0}$. By $l_{m}=l_{m}(x)$ we denote polyline with vertices $\left(\xi_{i}, y_{i}\right)$ $(i=0, \ldots, m)$. Since $l_{m}\left(\xi_{0}\right)=y_{0}=y_{m}=l_{m}\left(\xi_{m}\right)$, we can extend function $l_{m}(x)$ $2 \pi$-periodically and continuously to entire real axis. We will denote set of all such polygons as $\mathcal{L}_{m}$. Besides, it will be convenient to extend grid $\Omega_{m}=\left\{\xi_{i}\right\}_{i=0}^{m}$ in the following way: $\xi_{i+k m}=\xi_{i}+2 k \pi, k \in \mathbb{Z}$. Points $\xi_{i}$ divide real axis into segments

$$
\Delta_{i}=\left[\xi_{i}, \xi_{i+1}\right], \quad i \in \mathbb{Z} .
$$

On each segment function $l_{m}$ represents linear function

$$
l_{m}(x)=l_{m}^{i}(x)=\alpha_{i} x+\beta_{i}, \quad x \in \Delta_{i},
$$

where

$$
\alpha_{i}=\frac{y_{i+1}-y_{i}}{\xi_{i+1}-\xi_{i}}, \quad \beta_{i}=y_{i}-\alpha_{i} \xi_{i}, \quad i \in \mathbb{Z} .
$$

Let $S_{n}\left(l_{m}\right)=S_{n}\left(l_{m}, x\right)$ denote a partial sum of Fourier series for $l_{m}$ :

$$
S_{n}\left(l_{m}, x\right)=\frac{a_{0}}{2}+\sum_{k=1}^{n} a_{k} \cos k x+b_{k} \sin k x, \quad x \in \mathbb{R},
$$

where $a_{k}$ and $b_{k}$ are the Fourier coefficients of $l_{m}$ :

$$
a_{k}=\frac{1}{\pi} \int_{-\pi}^{\pi} l_{m}(t) \cos k t d t, \quad b_{k}=\frac{1}{\pi} \int_{-\pi}^{\pi} l_{m}(t) \sin k t d t .
$$


Also we should note the important special case of functions of this type. Let $f$ be a function from Sobolev space $W_{2 \pi}^{2,1}$. It means that $f$ is $2 \pi$-periodic function and $f^{\prime}$ is absolutely continuous on $[-\pi, \pi]$ and

$$
\int_{-\pi}^{\pi}\left|f^{\prime \prime}(t)\right| d t<\infty
$$

If we take $y_{i}=f\left(\xi_{i}\right)$, we get polygonal line $l_{m}$ which is inscribed in function $f$ :

$$
l_{m}\left(\xi_{i}\right)=f\left(\xi_{i}\right), \quad i \in \mathbb{Z} .
$$

We denote such a function as $l_{m}(f)=l_{m}(f, x)$. Set of all the polygonal lines on different grids inscribed in given function we denote as $L_{f}$.

The aim of this work is to study the approximation properties of partial Fourier sums of function $l_{m}$ or, in other words, estimate the value

$$
R_{n}\left(l_{m}\right)=R_{n}\left(l_{m}, x\right)=l_{m}(x)-S_{n}\left(l_{m}, x\right)
$$

both for arbitrary polygon and polygon inscribed in function $f$.

\section{Formulation of explicit form of $R_{n}\left(l_{m}\right)$}

According to Dirichlet principle [9; p.438] function $l_{m}$ can be expanded as uniformly convergent Fourier series:

$$
l_{m}(x)=\frac{a_{0}}{2}+\sum_{k=1}^{\infty} a_{k} \cos k x+b_{k} \sin k x, \quad x \in \mathbb{R} .
$$

From (0.2), (0.4) and (1.1) we have

$$
R_{n}\left(l_{m}, x\right)=l_{m}(x)-S_{n}\left(l_{m}, x\right)=\sum_{k=n+1}^{\infty} a_{k} \cos k x+b_{k} \sin k x, \quad n \geqslant 1 .
$$

Then, using partial integration method, from equalities (0.3) and (0.1) we obtain

$$
\begin{gathered}
a_{k}=\frac{1}{\pi} \int_{-\pi}^{\pi} l_{m}(t) \cos k t d t=\frac{1}{\pi k} \int_{-\pi}^{\pi} l_{m}(t) d \sin k t= \\
-\frac{1}{\pi k} \int_{-\pi}^{\pi} l_{m}^{\prime}(t) \sin k t d t=-\frac{1}{\pi k} \sum_{i=0}^{m-1} \int_{\xi_{i}}^{\xi_{i+1}}\left(l_{m}^{i}(t)\right)^{\prime} \sin k t d t= \\
-\frac{1}{\pi k} \sum_{i=0}^{m-1} \alpha_{i} \int_{\xi_{i}}^{\xi_{i+1}} \sin k t d t=\frac{1}{\pi k^{2}} \sum_{i=0}^{m-1} \alpha_{i}\left(\cos k \xi_{i+1}-\cos k \xi_{i}\right), \\
b_{k}=\frac{1}{\pi} \int_{-\pi}^{\pi} l_{m}(t) \sin k t d t=\frac{1}{\pi k^{2}} \sum_{i=0}^{m-1} \alpha_{i}\left(\sin k \xi_{i+1}-\sin k \xi_{i}\right) .
\end{gathered}
$$


Using the equation (1.3), and that $\alpha_{i}=\alpha_{i+m}$ and $\xi_{0}=-\pi, \xi_{m}=\pi$, we get

$$
\begin{gathered}
a_{k}=\frac{1}{\pi k^{2}}\left(\sum_{i=0}^{m-1} \alpha_{i} \cos k \xi_{i+1}-\sum_{i=0}^{m-1} \alpha_{i} \cos k \xi_{i}\right)=\frac{1}{\pi k^{2}} \sum_{i=1}^{m}\left(\alpha_{i-1}-\alpha_{i}\right) \cos k \xi_{i} \\
b_{k}=\frac{1}{\pi k^{2}} \sum_{i=1}^{m}\left(\alpha_{i-1}-\alpha_{i}\right) \sin k \xi_{i} .
\end{gathered}
$$

The last summand in (1.5) equals to zero, but we keep it in sum for convenience of further explanation. Using (1.4) and (1.5), we rewrite formula (1.2):

$$
R_{n}\left(l_{m}, x\right)=\frac{1}{\pi} \sum_{k=n+1}^{\infty} \frac{1}{k^{2}} \sum_{i=1}^{m}\left(\alpha_{i-1}-\alpha_{i}\right)\left(\cos k \xi_{i} \cos k x+\sin k \xi_{i} \sin k x\right) .
$$

Thence we obtain the following

LEMMA 1. For any $l_{m}$ from $\mathcal{L}_{m}$ and $L_{f}$ the following equality holds:

$$
R_{n}\left(l_{m}, x\right)=\frac{1}{\pi} \sum_{i=1}^{m}\left(\alpha_{i-1}-\alpha_{i}\right) \sum_{k=n+1}^{\infty} \frac{\cos k\left(x-\xi_{i}\right)}{k^{2}} .
$$

Let's proceed to the $R_{n}\left(l_{m}, x\right)$ estimation problem for $l_{m} \in \mathcal{L}_{m}$.

\section{Estimation of $R_{n}\left(l_{m}\right)$ for arbitrary polyline $l_{m} \in \mathcal{L}_{m}$}

The following theorem holds.

TheOREM 1. Let $l_{m} \in \mathcal{L}_{m}, 0<\varepsilon<\frac{1}{2} \min _{i \in \mathbb{Z}}\left|\xi_{i+1}-\xi_{i}\right|, \Delta_{i}^{\varepsilon}=\left[\xi_{i}+\varepsilon, \xi_{i+1}-\varepsilon\right]$. Then following inequalities are true:

$$
\begin{gathered}
\left|R_{n}\left(l_{m}, x\right)\right| \leqslant \frac{1}{\pi n} \sum_{i=1}^{m}\left|\alpha_{i-1}-\alpha_{i}\right|, \quad x \in \mathbb{R}, \\
\left|R_{n}\left(l_{m}, x\right)\right| \leqslant \frac{6}{\pi n^{2}} \sum_{i=1}^{m} \frac{\left|\alpha_{i-1}-\alpha_{i}\right|}{\left|\sin \frac{x-\xi_{i}}{2}\right|}, \quad x \in \Delta_{i}^{\varepsilon}, \quad i \in \mathbb{Z} .
\end{gathered}
$$

Proof 1. In order to prove inequality (2.1) we use lemma 1 and write

$$
\begin{aligned}
\left|R_{n}\left(l_{m}, x\right)\right| \leqslant & \frac{1}{\pi} \sum_{i=1}^{m}\left|\alpha_{i-1}-\alpha_{i}\right|\left|\sum_{k=n+1}^{\infty} \frac{\cos k\left(x-\xi_{i}\right)}{k^{2}}\right| \leqslant \\
& \frac{1}{\pi} \sum_{i=1}^{m}\left|\alpha_{i-1}-\alpha_{i}\right| \sum_{k=n+1}^{\infty} \frac{1}{k^{2}} .
\end{aligned}
$$

Because of

$$
\sum_{k=n+1}^{\infty} \frac{1}{k^{2}} \leqslant \int_{n}^{\infty} \frac{d x}{x^{2}}=\frac{1}{n}
$$


from (2.3) we obtain

$$
\left|R_{n}\left(l_{m}, x\right)\right| \leqslant \frac{1}{\pi n} \sum_{i=1}^{m}\left|\alpha_{i-1}-\alpha_{i}\right| .
$$

Thereby we proved that inequalities (2.1) are true.

To show (2.2) we separately estimate value

$$
A=\sum_{k=n+1}^{\infty} \frac{\cos k\left(x-\xi_{i}\right)}{k^{2}} .
$$

After applying Abel transformation we get

$$
A=\sum_{k=n+1}^{\infty}\left(\frac{1}{k^{2}}-\frac{1}{(k+1)^{2}}\right) \sum_{j=n+1}^{k} \cos j\left(x-\xi_{i}\right) .
$$

From above, and from easily obtained formula

$$
\sum_{k=m}^{n} \cos k x=\frac{\sin \frac{2 n+1}{2}-\sin \frac{2 m-1}{2}}{2 \sin \frac{x}{2}}
$$

we have

$$
|A| \leqslant \frac{1}{\left|\sin \frac{x-\xi_{i}}{2}\right|} \sum_{k=n+1}^{\infty} \frac{2 k+1}{k^{2}(k+1)^{2}} \leqslant \frac{6}{n^{2}\left|\sin \frac{x-\xi_{i}}{2}\right|} .
$$

From both the above inequality and first of inequalities in (2.3), we obtain

$$
\left|R_{n}\left(l_{m}, x\right)\right| \leqslant \frac{6}{\pi n^{2}} \sum_{i=1}^{m} \frac{\left|\alpha_{i-1}-\alpha_{i}\right|}{\left|\sin \frac{x-\xi_{i}}{2}\right|}, \quad x \in \Delta_{i}^{\varepsilon} .
$$

By that the inequality (2.2) and a theorem 1 are proved.

\section{Approximation of polygons $l_{m} \in L_{f}$ by Fourier sums}

We denote as $\mathcal{K}$ set of functions, that are constants on entire real axis, and let $\widetilde{W}_{2 \pi}^{2,1}=W_{2 \pi}^{2,1} \backslash \mathcal{K}$.

We'll consider a problem of approximation of polygons $l_{m} \in L_{f}$ when $f \in \widetilde{W}_{2 \pi}^{2,1}$. We prove the following

TheOREM 2. Let $f \in W_{2 \pi}^{2,1}, l_{m}(f) \in L_{f}$. Then the following estimate takes place:

where

$$
\left|l_{m}(f, x)-S_{n}\left(l_{m}(f), x\right)\right| \leqslant \frac{\kappa}{n},
$$

$$
\kappa=\frac{1}{\pi} \int_{-\pi}^{\pi}\left|f^{\prime \prime}(t)\right| d t
$$

and this estimate can't be improved in order, when $f \in \widetilde{W}_{2 \pi}^{2,1}$. In other words, there is positive constant $c=c(f)$, for which

$$
\sup _{l_{m} \in L_{f}} \max _{x}\left|l_{m}(f, x)-S_{n}\left(l_{m}(f), x\right)\right| \geqslant \frac{c}{n} .
$$


Proof 2. For function $l_{m}(f)$ the formula (1.6) also takes place. Besides, we note, that on each segment $\Delta_{i}=\left[\xi_{i}, \xi_{i+1}\right]$, according to Lagrange theorem, we can find value $t_{i}$, so that $f^{\prime}\left(t_{i}\right)=\alpha_{i}$. So, the expression (1.6) for $l_{m}(f)$ can be represented in the following form:

$$
R_{n}\left(l_{m}, x\right)=\frac{1}{\pi} \sum_{i=1}^{m}\left(f^{\prime}\left(t_{i-1}\right)-f^{\prime}\left(t_{i}\right)\right) \sum_{k=n+1}^{\infty} \frac{\cos k\left(x-\xi_{i}\right)}{k^{2}}, \quad t_{i} \in \Delta_{i} .
$$

Due to absolute continuity of $f^{\prime}$, the following takes place

$$
\left|f^{\prime}\left(t_{i-1}\right)-f^{\prime}\left(t_{i}\right)\right| \leqslant \int_{t_{i-1}}^{t_{i}}\left|f^{\prime \prime}(t)\right| d t .
$$

Also we can obtain the following estimate:

$$
\left|\sum_{k=n+1}^{\infty} \frac{\cos k\left(x-\xi_{i}\right)}{k^{2}}\right| \leqslant \frac{1}{n} .
$$

From (3.1), (3.2), (3.3) we have

$$
\left|R_{n}\left(l_{m}(f), x\right)\right| \leqslant \frac{1}{\pi n} \sum_{i=1}^{m} \int_{t_{j-1}}^{t_{j}}\left|f^{\prime \prime}(t)\right| d t=\frac{1}{\pi n} \int_{-\pi}^{\pi}\left|f^{\prime \prime}(t)\right| d t .
$$

We will show, that obtained estimate for $R\left(l_{m}(f)\right)$ can't be improved in order. Given that $f \not \equiv$ const, we can select $x_{0}$ in $(-\pi, \pi)$, so that $f\left(x_{0}\right) \neq f( \pm \pi)$. Let's build polygon $l_{2}(f, x)$ on the grid $\left\{-\pi, x_{0}, \pi\right\}$ and continue this polygon $2 \pi$-periodically on the entire real axis. From formula (1.6) and from $\alpha_{i}=\alpha_{i+2 k}, \quad k \in \mathbb{Z}$, we obtain

$$
R_{n}\left(l_{2}, x\right)=\frac{\alpha_{0}-\alpha_{1}}{\pi}\left(\sum_{k=n+1}^{\infty} \frac{\cos k\left(x-x_{0}\right)}{k^{2}}-\sum_{k=n+1}^{\infty} \frac{\cos k(x-\pi)}{k^{2}}\right) .
$$

We find lower bound for $R_{n}\left(l_{2}, x\right)$, for $x=x_{0}$.

$$
R_{n}\left(l_{2}, x_{0}\right)=\frac{\alpha_{0}-\alpha_{1}}{\pi}\left(\sum_{k=n+1}^{\infty} \frac{1}{k^{2}}-\sum_{k=n+1}^{\infty} \frac{\cos k\left(x_{0}-\pi\right)}{k^{2}}\right) .
$$

We find lower bound for the first sum and upper bound for the second. The first sum can be estimated using the integral:

$$
\sum_{k=n+1}^{\infty} \frac{1}{k^{2}}>\int_{n+1}^{\infty} \frac{d x}{x^{2}}=\frac{1}{n+1}
$$

To estimate second sum we use Abel transformation and get

$$
\sum_{k=n+1}^{\infty} \frac{\cos k\left(x_{0}-\pi\right)}{k^{2}}=\sum_{k=n+1}^{\infty} \frac{2 k+1}{k^{2}(k+1)^{2}} \sum_{i=n+1}^{k} \cos i\left(x_{0}-\pi\right) .
$$


Thence we deduce

$\left|\sum_{k=n+1}^{\infty} \frac{2 k+1}{k^{2}(k+1)^{2}} \sum_{i=n+1}^{k} \cos i\left(x_{0}-\pi\right)\right| \leqslant \sum_{k=n+1}^{\infty} \frac{2}{k^{2}(k+1)}\left|\sum_{i=n+1}^{k} \cos i\left(x_{0}-\pi\right)\right|=B$.

Using (2.4), we get

$$
B \leqslant \frac{2}{\left|\sin \frac{x_{0}-\pi}{2}\right|} \sum_{k=n+1}^{\infty} \frac{1}{k^{3}}<\frac{4}{n^{2}\left|\sin \frac{x_{0}-\pi}{2}\right|} .
$$

So, the remainder of Fourier sum for $l_{2}$ can be estimated in the following way:

$$
\left|R_{n}\left(l_{2}, x_{0}\right)\right| \geqslant \frac{1}{n+1}-\frac{4}{n^{2}\left|\sin \frac{x_{0}-\pi}{2}\right|} .
$$

We show, that the limit of ratio of upper and lower bounds equals some constant for given $f$ :

$$
\lim _{n \rightarrow \infty} \frac{\frac{1}{n+1}-\frac{4}{n^{2}\left|\sin \frac{x_{0}-\pi}{2}\right|}}{\frac{1}{\pi n} \int_{-\pi}^{\pi}\left|f^{\prime \prime}(t)\right| d t}=\frac{\pi}{\int_{-\pi}^{\pi}\left|f^{\prime \prime}(t)\right| d t}=c(f) .
$$

So, we proved, that the obtained estimate can't be improved in order.

\section{Список литературы}

[1] Walsh J.L., Ahlberg J.H., Nilson E.N. The Theory of Splines and Their Applications. Mathematics in Science and Engineering (Vol. 38), Academic Press, 1967. 283 p.

[2] Miroshnichenko V.L., Zav'yalov U.S., Kvasov B.I. Metody splain-funktsiy. Moscow: Nauka, 1980. 352 p. (in Russian)

[3] Subbotin U.N., Stechkin S.B. Splainy v vichislitel'noy matematike. Moscow: Nauka, 1976. 248 p. (in Russian)

[4] Sharapudinov I.I. Approximation properties of Fejer- and de la Vallee-Poussin-type means for partial sums of a special series in the system $\{\sin x \sin k x\}_{k=1}^{\infty} / /$ Sbornik: Mathematics, 2015. Vol. 206, Issue 4. Pp. 131-148.

[5] Zygmund A. Trigonometric Series, Vol. 1. Cambridge University Press, 2002. 747 p.

[6] Magomed-Kasumov M.G. Approximation properties of classic Vallee Poussin means for piecewise smooth functions // Herald of Daghestan Scientific Center, 2014. Vol. 54. Pp. 5-12. (in Russian)

[7] Magomed-Kasumov M.G. Approximation Properties of de la Vallee-Poussin Means for Piecewise Smooth Functions // Mathematical notes, 2016. Vol. 100. Issue 2. Pp. 229-247.

[8] Bari N.K. Trigonometricheskiye ryady. Moscow: FIZMATLIT, 1961. 936 p. (in Russian)

[9] Fihtengolts G.M. Kurs differentsial'nogo i integralnogo ischisleniya. Vol. 3. Moscow: FIZMATLIT, 2001. 728 p. (in Russian) 